\title{
Gesundheitsförderung und Prävention: wohin die Reise gehen soll
}

Christine Romanna,

Barbara Weil ${ }^{b}$

a Verantwortliche Ressort Gesundheitsförderung und Prävention

b Leiterin Abteilung Gesundheitsförderung und Prävention
Korrespondenz:

Dr. med. Christine Romann FMH / Ressort Gesundheit und Prävention

Elfenstrasse 18

CH-3000 Bern 15

Tel. 0313591111

Fax 0313591112
Bis 2020 will die FMH durch Gesundheitsförderung und Prävention einen entscheidenden Beitrag zu einer gesünderen Schweiz leisten: Das ist das Ziel, welches wir mit unserer neuen Strategie anvisieren. Um es zu erreichen, will die FMH eine aktive Rolle in der Gesundheitsförderung und Prävention übernehmen und die nötigen Aufgaben in den eigenen Reihen klären und verteilen. Unsere Mitglieder sollen vom Wert der Gesundheitsförderung und Prävention überzeugt sein und ihre ärztliche Kompetenz in alle Aktionsfelder einbringen. Ebenso beteiligen sie sich aktiv an der Konzeption und Umsetzung von entsprechenden Programmen.

So lässt sich in Kürze die neuausgearbeitete FMHStrategie des Ressorts Gesundheitsförderung und Prävention beschreiben, die im September 2011 den Delegierten zur Diskussion und Verabschiedung vorgelegt wird.

Blicken wir einen Moment zurück: Bereits Ende der 80er Jahre war die Prävention für die FMH präsent, HIV/Aids sowie Raucherentwöhnung waren dabei die drängendsten Fragen. Die FMH schaltete sich in die laufende Debatte zur Suchtpolitik ein, was in das noch heute überzeugende FMH-Drogenkonzept mündete. Eine Konsultativ-Kommission «Prävention und Gesundheitsförderung» wurde ins Leben gerufen - Vorläuferin der heutigen Präventionskommission. Zu Beginn der 90er Jahre wurde schliesslich die FMH-Abteilung «Prävention» ins Leben gerufen. Eine ihrer Aufgaben war die Konzipierung von Fortbildungsprogrammen in Zusammenarbeit mit dem Bundesamt für Gesundheit, das damals alle drei bis vier Jahre eine Präventionskampagne durchführte zu jeweils einem bestimmten Thema. Manche werden sich erinnern an Fortbildungsseminare wie «Krise und Suizid», oder «Back in Time», ein Projekt zur Früherfassung und besseren Behandlung von Lumbalgien. Nachdem der FMH-Zentralvorstand zu Beginn dieses Jahrhunderts beschlossen hat, sich auf die strategische Arbeit zu konzentrieren, hat sich die Abteilung in den folgenden Jahren zu einer Kompetenzstelle für alle gesundheitspolitischen Fragen rund um die Prävention entwickelt. Die FMH erhielt nach und nach Einsitz in viele wichtige nationale Gremien, wurde zunehmend in die entsprechenden Vernehmlassungen einbezogen und ist heute in allen Belangen der Gesundheitsförderung und Prävention eine gewichtige Partnerin.

Heute hat die Gesundheit sowohl für die einzelnen Menschen als auch für die Gesellschaft als Ganzes einen hohen Stellenwert. Entsprechend ist das Thema auch in der Politik präsent - wie die aktuellen Auseinandersetzungen rund um das Präventionsgesetz zeigen. Niemand bestreitet heute noch, dass viel getan werden kann und auch getan werden soll, um die Gesundheit zu erhalten und Krankheiten vorzubeugen. Ebenso ist mittlerweile gut belegt, dass sich Prävention auch finanziell auszahlt und hilft, die stetig ansteigenden Kosten zu dämpfen. Allerdings stehen auch handfeste wirtschaftliche Interessen auf dem Spiel, wenn Prävention ernst genommen wird man denke nur an die Geschichte der Tabakprävention. Während es im Parlament - zumindest vordergründig - um die Frage geht, welche Rolle der Staat in der Prävention einnehmen soll, stehen für die FMH andere Themen im Fokus: Wo setzen wir die Prioritäten im ganzen Feld von Gesundheitsförderung und Prävention, mit welchen Partnern wollen wir zusammenarbeiten, um das Ziel - eine gesündere Schweiz zu erreichen? Wie verteilen wir die anfallende Arbeit intern? Welche Fragen rund um Gesundheitsförderung und Prävention stellen sich in den verschiedenen Fachgebieten? Welche Projekte in den Kantonen oder Gemeinden erfordern unsere ärztliche Kompetenz und unser Engagement? Welchen Stellenwert sollen Gesundheitsförderung und Prävention in unserer täglichen Arbeit in der Praxis haben? Wie wird unsere Arbeit in diesem Bereich abgegolten? Und last, but certainly not least - was bedeutet Gesundheitsförderung und Prävention für den einzelnen Arzt, die einzelne Ärztin? Auch unsere eigene Gesundheit ist uns ein Anliegen!

Wir freuen uns auf eine angeregte Diskussion mit allen an Gesundheitsförderung und Prävention Interessierten. Wir hoffen, dass wir die Weichen in Richtung einer gesünderen Schweiz richtig gestellt haben, dass der Zug in den nächsten Monaten und Jahren auf Kurs bleibt - und gut in Fahrt kommt. 